\title{
Neonatal Late Onset Infection with Severe Acute Respiratory Syndrome Coronavirus 2
}

\author{
Danilo Buonsenso, MD ${ }^{1, *(1)}$ Simonetta Costa, $\mathrm{PhD}^{1, *}$ Maurizio Sanguinetti, PhD ${ }^{2}$ Paola Cattani, MD ${ }^{2}$ \\ Brunella Posteraro, MD ${ }^{2}$ Simona Marchetti, MD² Brigida Carducci, MD ${ }^{1}$ Antonio Lanzone, MD ${ }^{1}$ \\ Enrica Tamburrini, MD ${ }^{2}$ Giovanni Vento, MD $^{1}$ Piero Valentini, MD ${ }^{1}$
}

${ }^{1}$ Department of Woman and Child Health and Public Health, Fondazione Policlinico Universitario A. Gemelli IRCCS, Rome, Italy

2 Dipartimento Scienze di laboratorio e infettivologiche, Fondazione

Policlinico Universitario Agostino Gemelli IRCCS, Rome, Italy

Am J Perinatol 2020;37:869-872.
Address for correspondence Danilo Buonsenso, MD, Largo A. Gemelli 8, Rome 00168, Italy (e-mail: danilobuonsenso@gmail.com).

\begin{abstract}
Keywords

- SARS-CoV-2

- newborn

- COVID-19

- pregnancy

Objective To date, no information on late-onset infection in newborns to mother with severe acute respiratory syndrome coronavirus 2 (SARS-CoV-2) contracted in pregnancy are available. This study aimed to evaluate postdischarge SARS-CoV-2 status of newborns to mothers with COVID-19 in pregnancy that, at birth, were negative to SARS-CoV-2.

Study Design This is an observational study of neonates born to mothers with coronavirus disease 2019 (COVID-19).

Results Seven pregnant women with documented SARS-CoV-2 infection have been evaluated in our institution. One woman had a spontaneous abortion at 8 weeks of gestational age, four women recovered and are still in follow-up, and two women delivered. Two newborns were enrolled in the study. At birth and 3 days of life, newborns were negative to SARS-CoV-2. At 2-week follow-up, one newborn tested positive although asymptomatic.

Conclusion Our findings highlight the importance of follow-up of newborns to mothers with COVID-19 in pregnancy, since they remain at risk of contracting the infection in the early period of life and long-term consequences are still unknown.
\end{abstract}

\section{Key Points}

- Newborns to mothers with coronavirus disease 2019 (COVID-19) in pregnancy can acquire the infection later after birth.

- Newborns to mothers with COVID-19 in pregnancy need a long-term follow-up, even if they tested negative at birth.

- Specific guidelines for the long-term follow-up of newborns to mothers with COVID-19 in pregnancy are needed.

Since its first description, severe acute respiratory syndrome coronavirus 2 (SARS-CoV-2) has spread worldwide with over 2 million cases reported and thousands of deaths. Every human is susceptible to the infection, and pregnant

* These authors act as first authors. women are not spared. Although reports documented COVID-19 infections among pregnant women $^{1,2}$ and described the neonatal outcome relatively to early days of life, ${ }^{3}$ to date, no information on late-onset infection in newborns to mother with SARS-CoV-2 contracted in pregnancy are available.

Copyright $\odot 2020$ by Thieme Medical Publishers, Inc., 333 Seventh Avenue, New York, NY 10001, USA. Tel: +1(212) 760-0888.
DOI https://doi.org/ 10.1055/s-0040-1710541. ISSN $0735-1631$. 


\section{Materials and Methods}

In this observational study, neonates born to mothers with COVID-19 were included. This study was approved by the local ethics committee (prot ID 31404/2020). Informed consent was obtained from the neonates' parents.

\section{Results}

Seven pregnant women with documented SARS-CoV-2 infection have been evaluated in our institution. One woman had a spontaneous abortion at 8 weeks of gestational age, four women recovered and are still in follow-up, and two women delivered. Two newborns were enrolled in the study. The other women are currently followed-up in our center: one is admitted for preeclampsia with no respiratory symptoms (currently 35 weeks of gestational age), two women have been discharged home after having developed respiratory symptoms (currently at 20 and 27 weeks of gestational age, respectively), and one asymptomatic (currently at 18 weeks of gestational age). ${ }^{4}$

Clinical, microbiological, and epidemiological details of the two neonates are reported in -Fig. 1 and - Table 1.

Newborn 1 was born at $38^{3 / 7}$ weeks of gestational age, birth weight 3,390 g, and Apgar's scores was 9 to 10 . The neonate had no symptoms and was immediately quarantined in the pediatric unit. Real time-polymerase chain reaction (RT-PCR) tests on nasopharyngeal swabs on day 1 and 5 of life turned negative. At 15-day follow-up, the newborn was in good clinical conditions, birth weight was 4,080 g with normal clinical examination. The mother breastfed him at home. The newborn nasopharyngeal swab was positive, documenting a late-onset neonatal infection with SARSCoV-2.

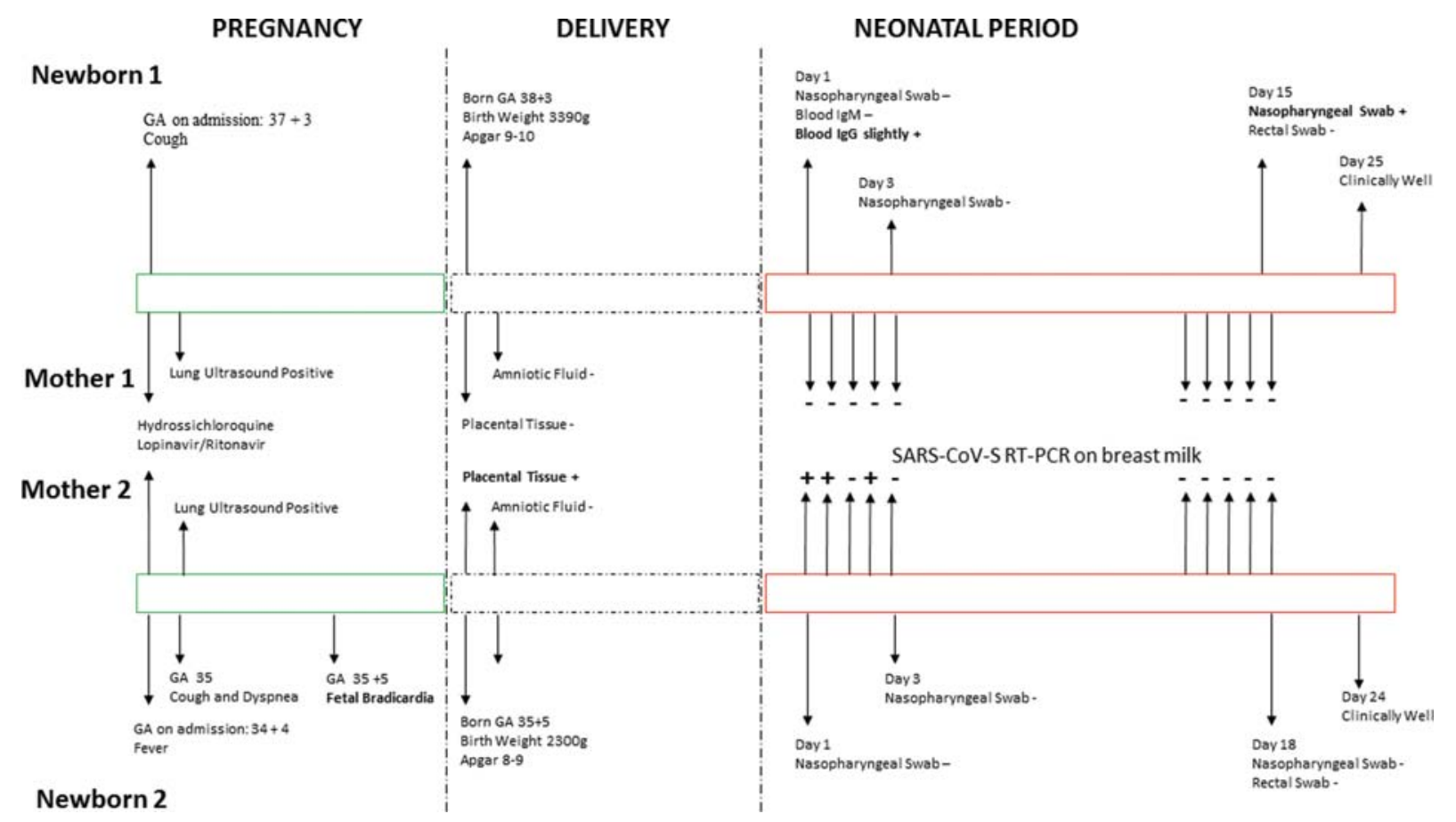

Fig. 1 Newborn 1: On March 19, 2020, a 42-year-old woman ( $37^{3 / 7}$ weeks of gestation) without known exposure to SARS-CoV-2 was admitted in our institution because of cough. Lung ultrasound showed diffuse interstitial lung disease. The RT-PCR on a nasopharyngeal swab was positive and treatment started. On March 26, an infant boy was delivered by cesarean section. His birth weight was 3,390 g and Apgar's scores were 9 to 10. The neonate had no symptoms and was immediately quarantined in the pediatric unit. On day 1 and 3 of life, RT-PCR tests on nasopharyngeal swabs turned negative. RT-PCR test on the placenta and umbilical blood were negative. The mother's breast milk tested with RT-PCR was result negative. The newborn was discharged on day 5 of life with the father who tested negative. On the same day, the mother was discharged and sent back home with a positive test. On day 15 of life, the newborn came back for the outpatient control. The newborn was healthy, with normal clinical examination. The mother decided to breastfeed him at home. Her breastmilk collected on day 11 to 14 had a negative RT-PCR test result. The newborn was tests with SARS-CoV-2 RT-PCR on nasal, pharyngeal, and rectal swab. The nasal swab resulted positive. Due to good conditions, the newborn was sent home with daily telemedicine updates to check his clinical status. Total 1 week later, the newborn is still in good clinical conditions. Newborn 2: On March 23, 2020, a 38-year-old woman (35/7 weeks of gestation)-exposed to both parents positive to SARS-CoV-2was admitted in our institution because of cough. Lung ultrasound showed diffuse interstitial lung disease. The RT-PCR on a nasopharyngeal swab was positive and treatment started. During hospital admission, the woman required low-flow oxygen therapy for 48 hours. On March 28 , due to fetal bradycardia, urgent cesarean section was performed. An infant boy was delivered by cesarean. His birth weight was $2,300 \mathrm{~g}$ and Apgar's scores were 8 to 9 . The neonate immediately quarantined in the neonatal subintensive unit. On day 1 and 3 of life, RT-PCR tests on nasopharyngeal swabs turned negative. RT-PCR test on the placenta and umbilical blood were positive. The mother's breast milk collected during the first 5 days of newborn life tested positive on 3 out of 5 samples for SARS-CoV-2 RT-PCR test. The newborn was discharged on day 13 of life with the mother and father, both negative on nasopharyngeal swabs. On day 18 of life, the newborn came back for the outpatient control. The newborn was healthy, his weight was $2,800 \mathrm{~g}$, with normal clinical examination. The father was feeding him with expressed breastmilk, which tested negative when collected on day 14 to 17 . The newborn was tested with SARS-CoV-2 RT-PCR on nasal, pharyngeal and rectal swab, all negative. RT-PCR, real time-polymerase chain reaction; SARS-CoV-2, severe acute respiratory syndrome coronavirus 2. 


\begin{tabular}{|c|c|c|}
\hline Variable & Newborn 1 & Newborn 2 \\
\hline Mother status & SARS-CoV-2 positive & SARS-CoV-2 positive \\
\hline Timing of maternal Infection & Third trimester & Third trimester \\
\hline Type of delivery & Cesarean section & Cesarean section \\
\hline Gestational age at delivery & $38^{3 / 7}$ weeks & $35^{5 / 7}$ weeks \\
\hline Birth weight & $3,390 \mathrm{~g}$ & $2,300 \mathrm{~g}$ \\
\hline In-hospital care & Mother/newborn separation & Mother/newborn separation \\
\hline In-hospital feeding & Formula & Formula \\
\hline Day of life when discharged & 5 & 13 \\
\hline Newborn status at discharge & SARS-CoV-2 negative blood IgG slightly positive & SARS-CoV-2 negative \\
\hline $\begin{array}{l}\text { Mother status when } \\
\text { discharged home }\end{array}$ & COVID-19 positive & COVID-19 negative \\
\hline $\begin{array}{l}\text { Father status when newborn } \\
\text { discharged home }\end{array}$ & COVID-19 negative & COVID-19 negative \\
\hline $\begin{array}{l}\text { Place where the newborn and } \\
\text { mother were discharged }\end{array}$ & Same household & Same household \\
\hline Household setting & $\begin{array}{l}\text { Newborn } 1 \\
\text { Mother (COVID-19 positive) } \\
\text { Father (COVID-19 negative) } \\
\text { Three brothers (not tested, asymptomatic) }\end{array}$ & $\begin{array}{l}\text { Newborn } 2 \\
\text { Mother (COVID-19 negative) } \\
\text { Father (COVID-19 negative) } \\
\text { One sister (not tested, asymptomatic) }\end{array}$ \\
\hline $\begin{array}{l}\text { Advice given to parents } \\
\text { on discharge }\end{array}$ & $\begin{array}{l}\text { Express breast milk to stimulate lactation } \\
\text { Avoid breastfeeding and breast milk at home } \\
\text { Father feeds the newborn with formula milk } \\
\text { Mother maintain a distance of at least } \\
6 \text { feet for as much of the time as possible } \\
\text { Hygiene suggestions }\end{array}$ & $\begin{array}{l}\text { Express breast milk } \\
\text { Father feeds the newborn with } \\
\text { expressed milk } \\
\text { Hygiene suggestions }\end{array}$ \\
\hline Feeding practice at home & $\begin{array}{l}\text { Breastfeeding with the mother } \\
\text { wearing a mask }\end{array}$ & $\begin{array}{l}\text { Father feeding the newborn with } \\
\text { expressed breast milk }\end{array}$ \\
\hline $\begin{array}{l}\text { Other parents with } \\
\text { COVID-19 not living in } \\
\text { the same household }\end{array}$ & None & $\begin{array}{l}\text { Grandfather COVID-19 positive } \\
\text { Grandmother COVID-19 positive }\end{array}$ \\
\hline Newborn outcome & $\begin{array}{l}\text { SARS-CoV-2 positive at } 15 \text { days of life } \\
\text { Clinically well }\end{array}$ & $\begin{array}{l}\text { SARS-CoV-2 negative at } 18 \text { days of life } \\
\text { Clinically well }\end{array}$ \\
\hline
\end{tabular}

Abbreviations: COVID-19, coronavirus 2019; RT-PCR, real time-polymerase chain reaction; SARS-CoV-2, severe acute respiratory syndrome coronavirus 2.

Notes: Newborn 1 was born at term and when discharged the mother was still positive.

Since the mother has three other children at home, she asked to be discharged at home respecting hygiene measures and wearing a mask, as well as the other indications given on discharge.

At home, she decided to breastfeed the newborn, which eventually turned SARS-CoV-2 positive on day 15 of life, although asymptomatic. Newborn 2 was born late-preterm and when discharged the mother was negative, as well the father, which was responsible to feed the newborn with expressed milk.

Newborn 2 was born at $35^{5 / 7}$ weeks of gestational age, birth weight 2,300 g, and Apgar's scores was 8 to 9 . He was immediately quarantined in the neonatal unit. RT-PCR tests on nasopharyngeal swabs on day 1 and 5 of life turned negative. At 18-day follow-up, the newborn was in good clinical conditions, birth weight was $2,800 \mathrm{~g}$ with normal clinical examination. The father fed him at home with expressed milk. The newborn nasopharyngeal and rectal swabs were negative.

\section{Discussion}

We demonstrated SARS-CoV-2 late-onset neonatal infection in a newborn to mother with confirmed COVID-19 in pregnancy.
The vertical infection was excluded by microbiological tests on the placenta, amniotic fluid, and two tests on the newborn.

Since evidences are still insufficient regarding the optimal perinatal care and no definitive guidelines are issued, ${ }^{5}$ risks/benefits analyses must be shared with the familydiscussing the choice of delivery, the impact and need of mother/newborn separation, the benefits of breastfeeding and its role in protecting newborns from infection, and factors influencing the postnatal risk of infection after discharge. ${ }^{6}$ In case 1 , the mother opted for breastfeeding. Since milk samples tested negative, respiratory secretions were the probable source of late-onset neonatal infection. However, the newborn was asymptomatic. We can speculate that the maternal immunoglobulin $\mathrm{G}$ (documented at birth 
in the neonatal blood) and breastmilk antibodies may have protected the newborn from a symptomatic infection, preserving the benefits of breastfeeding. The previous description of a 17-day-old newborn with COVID-19 pneumonia who contracted the infection via contact with his parents, who developed fever and cough after the newborn birth, would support our hypothesis that maternal antibodies might have protected our patient. ${ }^{7}$

Our report highlights the need of newborn follow-up even after the exclusion of vertical or early-onset neonatal infection with SARS-CoV-2, as happens with other viral infections acquired during pregnancy. To date, we do not know the long-term effects of SARS-CoV-2 infection acquired in the neonatal period. Our protocol include follow-up on day 15 and months 1, 3, 6, 9, and 12 of life. During each visit, the child undergoes clinical evaluation and nasopharyngeal/ rectal swabs, cerebral ultrasound is performed at months 1 to 6 (until the fontanel allows it), and visual and hearing tests are performed at months 6 to 12 .

Recently, Zeng et $\mathrm{al}^{3}$ reported a series of 33 newborns to mother with COVID-19, three of them being diagnosed with early-onset SARS-CoV-2 infection. Consistent with previous studies, ${ }^{3}$ the outcomes were good. However, these studies do not provide follow-up of these newborns.

\section{Conclusion}

Our findings highlight the importance of follow-up of newborns to mothers with COVID-19 in pregnancy, since they remain at risk of contracting the infection in the early period of life and long-term consequences are still unknown.

\section{Conflict of Interest}

None declared.

\section{References}

1 Chen H, Guo J, Wang C, et al. Clinical characteristics and intrauterine vertical transmission potential of COVID-19 infection in nine pregnant women: a retrospective review of medical records. Lancet 2020;395(10226):809-815

2 Yu N, Li W, Kang Q et al. Clinical features and obstetric and neonatal outcomes of pregnant patients with COVID-19 in Wuhan, China: a retrospective, single-centre, descriptive study. Lancet Infect Dis 2020; S1473-3099(20)30176-6. Doi: 10.1016/ S1473-3099(20)30176-6

3 Zeng L, Xia S, Yuan W, et al. Neonatal early-onset infection with SARS-CoV-2 in 33 neonates born to mothers with COVID-19 in Wuhan, China. JAMA Pediatr 2020. Doi: 10.1001/jamapediatrics.2020.0878

4 Buonsenso D, Raffaelli F, Tamburrini E, et al. Clinical role of lung ultrasound for the diagnosis and monitoring of COVID-19 pneumonia in pregnant women. Journal of Ultrasound in Obstetrics Gynecology. In presso; Available at: https://obgyn.onlinelibrary. wiley.com/doi/full/10.1111/aogs.13836. Accessed April 22, 2020

5 Chandrasekharan P, Vento M, Trevisanuto D, et al. Neonatal resuscitation and postresuscitation care of infants born to mothers with suspected or confirmed SARS-CoV-2 infection. Am J Perinatol 2020. Doi: 10.1055/s-0040-1709688

6 Puopolo KM, Hudak ML, Kimberlin DW, Cummings J. Initial guidance: management of infants born to mothers with COVID-19. American Academy of Pediatrics Committee on Fetus and Newborn, Section on Neonatal Perinatal Medicine, and Committee on Infectious Diseases Available at: https://downloads.aap.org/AAP/PDF/ COVID\%2019\%20Initial\%20Newborn\%20Guidance.pdf

7 Zeng LK, Tao XW, Yuan WH, Wang J, Liu X, Liu ZS. [First case of neonate infected with novel coronavirus pneumonia in China]. Zhonghua Er Ke Za Zhi 2020;58(00):E009 